\title{
SUB-POISSONIAN STATISTICS AS AN EXPERIMENTAL TEST FOR THE CONTEXTUALITY OF QUANTUM THEORY
}

\author{
D. DIEKS, H.F. ARNOLDUS and G. NIENHUIS \\ Fysisch Laboratorium, Rijksuniversiteit Utrecht, Postbus 80.000, 3508 TA Utrecht, The Netherlands
}

Received 25 April 1984

\begin{abstract}
It is argued that the phenomenon of sub-poissonian statistics can be regarded as experimental evidence for the contextual character of quantum theory. To this end, it is shown that the statistics predicted by non-contextual hidden-variable theories must satisfy certain inequalities which are a kind of local counterparts of the famous Bell inequalities and which exclude sub-poissonian statistics.
\end{abstract}

1. Introduction. Most recent work on the interpretation of quantum mechanics hinges on the question whether local hidden-variable theories are possible which reproduce the statistical predictions of quantum mechanics. The issue is amenable to experimental investigation since such local hidden variable theories must statisfy inequalities of the Bell-type, whereas it can be shown that the quantum mechanical formalism leads to predictions which, in certain circumstances, violate those inequalities. The experimental results [1] thus far strongly favour quantum mechanics; i.e. it seems that experiment excludes the validity of any local hidden-variable theory.

The common emphasis on the importance of the notion of locality tends to obscure the fact that a more general point is at issue here. In a number of socalled no-hidden-variable-proofs by Gleason [2], Kochen and Specker [3], and others [4], it has been demonstrated that the predictions of quantum mechanics are incompatible with non-contextual hidden variable theories, i.e. theories in which the result of an individual measurement of a physical quantity is independent of which other physical quantities are measured along with it. The proofs by Gleason and by Kochen and Specker show that it is already impossible to fulfil the requirement that the measurement results do not depend on which other physical quantities are also measured if these other quantities correspond to quantum mechanical observables commuting with the observable representing the original measurement. It remains essential for the proof, however, that not all these observables commuting with the first one commute among each other. In fact, the Bell inequalities can be regarded as an illustration of the same basic point. This follows from the circumstance that the distance between the positions where measurements are performed does not enter explicitly into the inequalities or their derivation. This means that the inequalities are also valid for the correlations between physical quantities measured at the same position; but now the "locality condition", according to which a measurement outcome depends only on the state of the object and the state of the measurement apparatus which is used for that particular measurement, becomes a "non-contextuality condition", Quantum mechanics furnishes predictions which can violate such "local Bell inequalities" if the physical quantities which are involved are represented by operators $A_{1}$, $A_{2}, B_{1}, B_{2}$ such that

$$
\begin{aligned}
& {\left[A_{i}, B_{j}\right]=0, \quad i, j=1,2,} \\
& {\left[A_{1}, A_{2}\right] \neq 0, \quad\left[B_{1}, B_{2}\right] \neq 0 .}
\end{aligned}
$$

Of course, the physical significance of these results is particularly clear if there is a space-like separation between the measurements, because then the non-contextuality cannot be accounted for by some physical disturbance which propagates from one measurement 
to another. This explains the care which has been taken in the most recent experiments to exclude any physical interaction between the two pieces of measurement apparatus. But, given that these experiments verify the quantum mechanical predictions while virtually excluding any mutual influence between the measurement set-ups, and furthermore given that there is no theoretical basis for assuming such interactions, it seems worth-while to point out that the conflict between quantum theoretical predictions and the assumption of non-contextual hidden variables can also be demonstrated experimentally in the context of local experiments.

In this letter we propose a method for bringing out, in an experimental way, the difference between quantum theory and non-contex tual hidden variable theories of the field type. It turns out that it is only necessary to consider one single dynamical variable (albeit at different times) so that the experimental test is at least conceptually - very simple. In fact, experimental results are already available in the literature. These results confirm the quantum mechanical predictions and contradict non-contextual hidden variable models from a certain class of field theories to be specified below. The principle of the test can readily be generalized to the case of two observables that are measured at different positions.

\section{Inequalities for non-contex tual hidden variable}

theories. Consider a physical system which is described, according to some non-contextual hidden variable theory, by a set of hidden parameters $\lambda_{1}, \lambda_{2} \ldots, \lambda_{k}$. For convenience we shall indicate this set by a single $\lambda$. During a time interval $[0, T]$ a measuring device is operating in such a way that the result of the measurement consists in a number of discrete detection events. In other words, the output of the detector can be described as a so-called "dot process" [5]. A prime example is a photodetector that records photo-electric pulses with a probability proportional to the intensity of the incident field. In general, the device may be a counter which has an activation probability that is a function of the incoming signal. This probability will depend on $\lambda_{t}$, but not on the outcomes at other times $t^{\prime} \neq t$. The latter follows from the assumption of noncontextuality and the supposed ideality of the measuring device (which does not disturb the passing signal). We shall now suppose that there is a continuous prob- ability density $p(t)$ such that $p(t) \Delta t$ is the probability that the device produces a "dot" on the time axis within a time interval of length $\Delta t$ around $t$ (for $\Delta t$ $\rightarrow 0$ ). In view of the above assumptions we have that $p(t)$ is a function of $\lambda_{t}$ :

$p(t)=f_{1}\left(\lambda_{t}\right)$,

whereas repetitions of the experiment correspond with some distribution $\rho(\lambda)$ over $\lambda$. Let $N$ denote the number of detected pulses during the counting interval.

Repetition of the experiment leads to a statistical distribution of $N$. The theory of random events [5] gives the factorial moments of this distribution as multiple integrals of the joint probability distribution functions:

$\langle N\rangle=\int \mathrm{d} \lambda \rho(\lambda) \int_{0}^{T} f_{1}\left(\lambda_{t}\right) \mathrm{d} t$,
$\langle N(N-1)\rangle=\int \mathrm{d} \lambda \rho(\lambda) \int_{0}^{T} \int_{0}^{T} f_{2}\left(t_{1}, t_{2}\right) \mathrm{d} t_{1} \mathrm{~d} t_{2}$,

etc. The function $f_{2}\left(t_{1}, t_{2}\right)$ has the following physical meaning: $f_{2}\left(t_{1}, t_{2}\right) \mathrm{d} t_{1} \mathrm{~d} t_{2}$ is the probability that in both the intervals $\left(t_{1}, t_{1}+\mathrm{d} t_{1}\right)$ and $\left(t_{2}, t_{2}+\mathrm{d} t_{2}\right)$ a random event is recorded, regardless of what happens outside these intervals. In view of the assumption of non-contextuality the function $f_{2}\left(t_{1}, t_{2}\right)$ factorizes according to

$f_{2}\left(t_{1}, t_{2}\right)=f_{1}\left(\lambda_{t_{1}}\right) f_{1}\left(\lambda_{t_{2}}\right)$.

This illustrates that $N$ obeys a Poisson distribution if a single $\lambda_{t}$ is reproduced in repetitions of the experiment. In the more general case of a distribution $\rho(\lambda)$, we find from

$$
\int \mathrm{d} \lambda \rho(\lambda)\left(\int_{0}^{T}\left[f_{1}\left(\lambda_{t}\right)-\left\langle f_{1}\left(\lambda_{t}\right)\right\rangle\right] \mathrm{d} t\right)^{2} \geqslant 0,
$$

that the following inequality holds:

$\langle N(N-1)\rangle-\langle N\rangle^{2} \geqslant 0$.

Inequality (5) pertains to the counting statistics of one single detector. However, it is easy to obtain generalizations for the case of two (or more) counters. For instance, for two counters with response functions $f_{1}(\lambda)$, $g_{1}(\mu)$ and with $\Delta f(\lambda, \mu) \equiv f_{1}(\lambda)-g_{1}(\mu)$, we find from 
$\int \mathrm{d} \lambda \mathrm{d} \mu \rho(\lambda, \mu)\left(\int_{0}^{T}[\Delta f-\langle\Delta f\rangle]\right)^{2} \geqslant 0$,

the inequality

$\left\langle\left(N_{1}-N_{2}\right)^{2}-\left(N_{1}+N_{2}\right)\right\rangle-\left\langle N_{1}-N_{2}\right\rangle^{2} \geqslant 0$.

For the case of photodetection signals produced by classical electromagnetic fields this inequality can also be found in ref. [6].

\section{Predictions of quantum theory and experimental} results. The above inequalities are not derivable, in general, from the quantum formalism. This is due to the fact that probabilities can no longer be represented by functions of an external field. Instead, we encounter expressions like $[7,8]$

$f_{2}\left(t_{1}, t_{2}\right)=\operatorname{Tr}\left\{W F^{-}\left(t_{1}\right) F^{-}\left(t_{2}\right) F^{+}\left(t_{2}\right) F^{+}\left(t_{1}\right)\right\}$,

for the joint probability distribution that a detector which operates by absorption is activated at $t_{1}$ and $t_{2}$. Here $F^{-}$and $F^{+}$are the negative and positive frequency parts of the operator representing the quantized field (in the Heisenberg picture) and $W$ is the density operator of the system. Since the field operators $F^{ \pm}$ do not commute, we cannot write this probability density in the form $f_{1}\left(\lambda_{t_{1}}\right) f_{1}\left(\lambda_{t_{2}}\right)$ unless $W$ possesses special properties. A quite analogous situation occurs in the case of the Bell inequalities. For instance, in the correlated spin experiment (the thought experiment of Einstein, Podolsky and Rosen in Bohm's version) classical relations obtain if the quantum system is described by a diagonal mixture of the form

$$
\int \mathrm{d}^{3} x p(x)\left|x_{1}^{+}\right\rangle\left|x_{2}^{-}\right\rangle\left\langle x_{2}^{-}\right|\left\langle x_{1}^{+}\right|,
$$

$p(x) \geqslant 0$, with $x$ a direction in space and $\left|x^{+}\right\rangle,\left|x^{-}\right\rangle$ eigenstates of the spin operator in the direction $x$ of the two spin $\frac{1}{2}$ particles. Similarly, we retrieve the classical expression from eq. (8) if $W$ is a mixture of eigenstates of the operator $F^{+}$. For the case of electromagnetism, this corresponds to the well-known Glauber representation [8] $\int \mathrm{d}^{2} \alpha P(\alpha)|\alpha\rangle\langle\alpha|$ in terms of coherent states $(\alpha \in \mathrm{C}, P(\alpha) \geqslant 0)$.

In general, however, quantum mechanical predictions will violate inequalities (5) and (7). A case in point is the fluorescence radiation field emitted by a two-state atom in a laser field. This case has been studied extensively by Mandel [9], Lenstra [10] and by
Arnoldus and Nienhuis [11]. If $N$ stands for the number of photons detected by a photon absorption counter (like a photomultiplier) during the interval $[0, T]$ and if the following definition is introduced:

$Q \equiv \frac{\langle N(N-1)\rangle-\left\langle N^{2}\right\rangle}{\langle N\rangle}$,

these workers find that $Q$ can assume negative values; the predicted minimum value of $Q$ in the steady state is $-\frac{3}{4}$ (of course, $Q$ has an absolute lower bound -1 ). For the classical case it follows from inequality (5) that $Q \geqslant 0$; note that for Poisson statistics $Q=0$. Statistics with $Q<0$ are called sub-poissonian. Arnoldus and Nienhuis [12] have also investigated the correlations between photons from the two sidebands in the three-line fluorescence spectrum. Introducing

$Q_{\mathrm{c}} \equiv \frac{\left\langle\left(N_{1}-N_{2}\right)^{2}-\left(N_{1}+N_{2}\right)\right\rangle-\left\langle N_{1}-N_{2}\right\rangle^{2}}{\left\langle N_{1}+N_{2}\right\rangle}$

as a measure for the correlation between the photon detections in two of the three lines, they find that $Q_{\mathrm{c}}$ can approach the absolute minimum value -1 arbitrarily close when $T \rightarrow \infty$. Here also, we have $Q_{\mathrm{c}} \geqslant 0$ for classical fields [inequality (7)] and $Q_{\mathrm{c}}=0$ for Poisson statistics.

Experimental observation of sub-poissonian statistics in fluorescence radiation has recently been reported by Short and Mandel [13]. They find a value of $Q=-0.00252 \pm 0.00040$, which provides statistically significant evidence for a sub-poissonian distribution and which is in agreement with the theoretically expected value of $Q$ for the case in question, $Q \approx$ -0.0023 .

4. Discussion of the significance of the results. The interpretation of violations of the inequalities (5) and (7) is not so simple as might seem the case on the basis of the foregoing exposition. The reason is that an important assumption has been made in the derivation of eq. (2b). This equation is only valid [5] if the function $f_{2}\left(t_{1}, t_{2}\right)$ does not possess the characteristics of the data function $\delta\left(t_{1}-t_{2}\right)$ for $t_{1} \rightarrow t_{2}$. The assumption in question has been made through the stipulation that $p(t)$ in eq. (1) be a continuous probability distribution. Physically speaking, the supposition is that the probability density that two counts are recorded within the time interval $\Delta t$ vanishes as $\Delta t \rightarrow 0$. This assumption 
is natural (and commonly made) if the measuring device responds to a continuous incoming field (for instance, and electromagnetic field). In the case that distinct particles are coming in, however, the assumption clearly is unwarranted. Localized particles can follow each other within arbitrarily small time intervals; as a consequence, there is a positive contribution to the integral in eq. (2b) from the values $t_{1}, t_{2}$ such that $t_{1} \approx t_{2}$. To see what happens, let us suppose that the counter responds to an incoming field with particlelike properties in such a way that

$f_{1}\left(\lambda_{t}\right)=a \sum_{i=1}^{M} \delta\left(t-\tau_{i}\right)+b\left(\lambda_{t}\right)$

with $b\left(\lambda_{t}\right)$ a continuous function of $t$. We then find:

$$
\begin{aligned}
& \left\langle f_{2}\left(t_{1}, t_{2}\right)\right\rangle=\int \mathrm{d} \lambda \rho(\lambda) f_{2}\left(t_{1}, t_{2}\right)=g_{2}\left(t_{1}, t_{2}\right) \\
& +a^{2} \delta\left(t_{1}-t_{2}\right) \int \mathrm{d} \lambda \rho(\lambda) \sum_{i=1}^{M} \delta\left(t_{1}-\tau_{i}\right)
\end{aligned}
$$

where $g_{2}\left(t_{1}, t_{2}\right)$ is continuous for $t_{1} \rightarrow t_{2}$. Integration over $t_{1}$ and $t_{2}$ gives:

$$
\int_{0}^{T} \int_{0}^{T} \mathrm{~d} t_{1} \mathrm{~d} t_{2}\left\langle f_{2}\left(t_{1}, t_{2}\right)\right\rangle=\langle N(N-1)\rangle+a^{2}\langle M\rangle
$$

This equation takes the place of eq. (2b), so that inequality (5) is no longer derivable. In the extreme case where $a=1$ and $b\left(\lambda_{t}\right) \equiv 0$, we find instead of inequality (5) the trivial inequality

$$
\left\langle N^{2}\right\rangle-\langle N\rangle^{2} \geqslant 0 \text {. }
$$

This result is intuitively obvious, as it corresponds to an ideally efficient counter which detects incoming particles. An arbitrary statistical distribution of detection times can evidently be obtained by judicious choice of the emission times of the particles that are to be detected.

The situation can be viewed from a slightly different angle by a consideration of what happens if the counter possesses a dead time $\tau_{K}$. The counting interval $[0, T]$ can be divided into $K$ intervals of length $\tau$, $T=K \tau$. For simplicity, consider the schematic case in which interval $l$ possesses a probability $p_{l}$ of showing up a count. We then have for the variance and mean value of $N$ :

$$
\sigma^{2}=\sum_{l=1}^{K} p_{l}\left(1-p_{l}\right), \quad \mu=\sum_{l=1}^{K} p_{l}
$$

so that

$$
\langle N(N-1)\rangle-\langle N\rangle^{2}=\sigma^{2}-\mu=-\sum_{l=1}^{K} p_{l}^{2} .
$$

We see from this that the presence of a dead time of the counter tends to make $\sigma^{2}-\mu$ negative; in experimental tests of the inequalities (5) and (7) this effect must be taken into account. To pass to the ideal case discussed before, we should take the limit for $\tau \rightarrow 0$, $K \rightarrow \infty, T$ constant, $\mu=\Sigma_{l=1}^{K} p_{l}$ constant. A necessary condition that $\sigma^{2}-\mu$ vanishes in this limit is that $\lim p_{l}=0$. This excludes the case of localized particles; for such particles the probability to be detected may remain positive in an arbitrary narrow time interval.

On the basis of the above remarks the significance of violations of inequalities (5) and (7) can be assessed as follows. If it is assumed that a theoretical model of the situation should be couched in terms of continuous fields and continuous probability densities, violation of the inequalities demonstrates that a non-contextual hidden variable model which predicts the correct counting statistics is not possible. In this case the inequalities (5) and (7) are on a par with the Bell inequalities. The crucial point of the Bell inequalities is that they express the limits set to the correlation between two series of measurement outcomes if this correlation is "transported" by a classical signal. In exactly the same way inequalities (5) and (7) set a bound to the correlation between successive measurement outcomes if these outcomes (and their correlations) all depend on an external classical signal. Quantum mechanics is able to predict stronger correlations than classical theory because of the mechanism of "wave packet reduction" by which the state description is changed depending on a measurement result.

However, violation of inequalities (5) and (7) could also be taken as merely a reflection of the particlelike character of the incoming beam. One could argue that the verification of sub-poissonian statistics is also relevant in this case: although it is well known that classically continuous fields take on particle-like properties in a quantum description, it is rather difficult 
to find clear-cut experimental evidence for this.

But this second interpretation of violations of the inequalities is by no means always the natural one in the context of quantum mechanics. For instance, in the case of photon statistics, for which the sub-poissonian character of the statistics has been verified experimentally, it seems rather dubious whether the second interpretation can be reconciled with quantum theory. Indeed, it is denied in the quantum theoretical description that photons can be localized. It therefore appears more natural to regard the pertinent experimental results as a reflection of the contextual character of quantum mechanics.

\section{References}

[1] A. Aspect, J. Dalibard and G. Roger, Phys. Rev. Lett. 49 (1982) 1804.

[2] A.M. Gleason, J. Math. Mech. 6 (1957) 885.

[3] S. Kochen and E.P. Specker, J. Math. Mech. 17 (1967) 59.
[4] F.J. Belinfante, A survey of hidden variable theories (Pergamon, London, 1973).

[5] N.G. van Kampen, Stochastic processes in physics and chemistry (North-Holland, Amsterdam, 1981).

[6] J. Dalibard and S. Reynaud, Non-classical properties of resonance fluorescence light, to be published in: New trends in atomic physics, Proc. Les Houches Summer School (July 1982), eds. G. Grynburg and R. Stora (North-Holland, Amsterdam, 1984).

[7] P.L. Kelley and W.H. Kleiner, Phys. Rev. A136 (1964) 316.

[8] R.J. Glauber, Optical coherence and photon statistics, in: Quantum optics and electronics, eds. C. de Witt, A. Blandin and C. Cohen-Tannoudji, (Gordon and Breach, New York, 1965).

[9] L. Mande1, Opt. Lett. 4 (1979) 205.

[10] D. Lenstra, Phys. Rev. A26 (1982) 3369.

[11] H.F. Arnoldus and G. Nienhuis, Opt. Acta 30 (1983) 1573.

[12] H.F. Arnoldus and G. Nienhuis, Opt. Commun. 48 (1984) 322 .

[13] R. Short and L. Mandel, Phys. Rev. Lett. 51 (1983) 384. 